\title{
Family Therapy and Medical Issues
}

\author{
Maria Cristina Canavarro • Frank M. Dattilio
}

Published online: 20 April 2011

(C) Springer Science+Business Media, LLC 2011

\section{Introduction}

Family therapists throughout the world are increasingly challenged by couples and families with medical conditions and physical complications (Law et al. 2000; McDaniel et al. 1992). Research has demonstrated that health matters and life-threatening diseases often have a unique impact on the dynamics of the marital relationship and/or family functioning (Rolland 1994; Walsh and Anderson 1988). Conversely, it also has been suggested that marital and family relationships can affect health in numerous ways (Fisher 2006; Weihs et al. 2002). From a family systems perspective, it is quite arduous to separate the effect that marital and family relationships have on a particular disease from the effect of the disease on the marital and family relationships (Burman and Margolin 1992). The dynamics are often woven into a mosaic of complexity that is resistant to change.

Therefore, special attention must be given to the particular issues that family therapists face when embarking on such challenging cases. Many questions often arise with these types of cases, such as whether or not a therapist can adequately assist a patient with a particular disease without considering the potential effects on other family members, and whether it is possible to help these patients without including both the partner and/or other family members and the interdependence that is inherent to such entanglement. These are just some of the many important questions that a therapist needs to consider when intervening with a patient, a couple, or a family challenged by any type of medical condition.

Despite the relevance of such questions, much of the professional literature has focused on health issues and illnesses from an individual point of view with less emphasis given to the impact of the disease on the marital and family dynamics (Ramsey 1989). Unarguably, a disease experienced by one family member can influence the family as a whole (Broderick 1993; Rolland 1994). For example, spouses and family members often contribute directly or

M. C. Canavarro $(\bowtie)$

Universidade de Coimbra, Coimbra, Portugal

e-mail: mccanavarro@fpce.uc.pt

F. M. Dattilio

Harvard Medical School, Boston, MA, USA

e-mail: Frankdattilio@cs.com 
indirectly to the appearance of symptoms and also can influence the adaptation to the disease, treatment decisions, and the participation in rehabilitation. The disease itself also may influence patterns of family communication, family cohesion, closeness, and family roles, among other aspects, which in turn may have a significant effect on a patient's adjustment to the illness (Cordova et al. 2001; Lepore et al. 2000). Living with a chronic disease, such as cancer or HIV, or another medical issue, as well as caring for a family member with a chronic disease can lead to physical and emotional stress. Some of the studies conducted in the area of Alzheimer and cancer patients, along with their caregivers, have shown that the caregiver's loss of personal freedom and restriction of social activities are associated with symptoms of emotional distress (Cairl and Kosberg 1993), including depression, frustration, and resentment (Skaff and Pearlin 1992), not to mention caregiver burden (Nijboer et al. 1998). Indeed, the diagnosis of a disease, particularly a life-threatening disease, can have a significant impact upon all family members, potentially affecting the overall dynamics of the relationships.

This special issue has been inspired by the increasing number of researchers interested in investigating the influence of medical diseases on intimate relationships, as well as the influence of intimate relationships on medical diseases (Campbell 1986). The contents are specifically dedicated to addressing some pertinent questions related to couples and families that influence and are influenced by medical diseases. Underlying the majority of these studies are the social policies of Western societies that were proposed in the beginning of the twenty-first century. They generally highlight: the urgency of specific actions to increase efforts related to multilevel prevention of disease and disability; the assessment of patients' health perceptions in order to effectively tailor treatment approaches to their needs; and the development of individual, family, and community resources, which may increase the quality of actual global health systems.

In this issue we have selected a number of studies that were conducted through the Universidade de Coimbra in Portugal. These studies clearly reflect some of the emerging health topics of concern in other developed Western countries. In brief, the studies presented here illustrate how family therapy research and practice may constitute an effective tool to address important psychosocial variables in a variety of relational and medical contexts. The lead article, "Congruence of the Marital Relationship during Transition to Parenthood: A Study with Couples who Conceived Spontaneously or through Assisted Reproductive Technologies" by Sofia Gameiro, Mariana Moura-Ramos, Maria Cristina Canavarro, Teresa Almeida-Santos and Frank Dattilio, addresses the marital relationship and satisfaction in couples conceiving through assisted technologies. This is a very recent medical procedure that was legislated in Portugal in 2006, and has been, or will be soon available in most developed Western countries.

The second article, "Ecological Contexts in Adolescent Pregnancy: The Role of Individual, Sociodemographic, Familial and Relational Variables in Understanding Risk of Occurrence and Adjustment" by Anabela Pedrosa, Raquel Pires, Paula Carvalho, Maria Cristina Canavarro and Frank Dattilio, addresses the adjustment of adolescent mothers in relation to their family and social contexts. Portugal has systematically reported elevated rates of teenage pregnancy, which are also observable (though in much higher incidences) in the United States and the United Kingdom, as well as in some of the most recently created European nations (i.e., Slovakia, Estonia, Hungary). This is followed by the article titled "Amniocentesis Due to Advanced Maternal Age: The Role of Marital Intimacy in Couples Decision-Making Process" by Bárbara Nazaré, Ana Fonseca, Sofia Gameiro, Maria Cristina Canavarro and Frank Dattilio, which focuses on couple functioning 
in situations of late pregnancy, whose prevalence tends to increase in modern societies where financial achievement and work production assume significant proportions.

An additional study, "Couple-Focused Interventions for HIV-Serodiscordant Couples during Transition to Motherhood" by Marco Pereira, Frank Dattilio, Maria Cristina Canavarro and Isabel Narciso, addresses therapeutic couple-focused strategies that may be outlined for serodiscordant spouses facing immediate reproductive decisions and a number of future uncertainties following the diagnosis of HIV infection in women during prenatal examinations. This is one of the most common situations in contemporary society in which a woman becomes aware of an HIV condition.

The interrelation between individual adjustment, marital relationship, and body image among breast cancer patients in two different phases of the disease is the highlight of the next article, "Marital Relationship, Body Image and Psychological Quality of Life among Breast Cancer Patients: The Moderating Role of the Disease's Phases" by Helena Moreira, Carla Crespo, Tiago Paredes, Sónia Silva, Maria Cristina Canavarro and Frank Dattilio. This is the most prevalent type of cancer among women in the United States and other Western countries, such as Portugal. The research questions that were explored in this study deserve greater attention in the professional literature as the interrelation between these variables has been scarcely investigated, particularly among Portuguese patients, despite their relevance for both research and clinical practice.

The final article, "Understanding Quality of Life in Children with Asthma and their Parents: Family Resources and Challenges" by Carla Crespo, Carlos Carona, Neuza Silva, Maria Cristina Canavarro and Frank Dattilio, focuses on the involvement of family caregivers in treatment routines of pediatric asthma (the most common childhood medical/ chronic health condition in developed countries) in order to promote treatment efficacy, reduce human burden, and prevent healthcare overutilization.

Although this series of studies was conducted in Portugal, it is our firm belief that many of the medical complications and familial struggles are axiomatic and can easily be found in most cultures throughout the world. All of these studies depict different clinical scenarios and portray the manner in which relational variables affect and are affected by medical conditions. They outline some implications and guidelines for couple and family interventions and what therapists need to know, particularly when encountering such challenging cases. They also represent a valuable contribution for the enrichment of family therapy because of their focus on medical settings that have emerged and/or have acquired greater prominence in recent decades and on which research in the family context is still recent. Moreover, the emphasis given to these modern medical settings can facilitate the achievement of the goals underlying contemporary Western health policies.

\section{References}

Broderick, C. (1993). Understanding family processes: Basics of family systems theory. Thousand Oaks, CA: Sage Publications, Inc.

Burman, B., \& Margolin, G. (1992). Analysis of the association between marital relationships and health problems: An interactional perspective. Psychological Bulletin, 112(1), 39-63.

Cairl, R., \& Kosberg, J. (1993). The interface of burden and level of task performance in caregivers of Alzheimer's disease patients: An examination of clinical profiles. Journal of Gerontological Social Work, 19, 133-151.

Campbell, T. (1986). Family's impact on health: A critical review. Family Systems Medicine, 4(2-3), 135-328. 
Cordova, M., Cunningham, L., Carlson, C., \& Andrykoswki, M. (2001). Social constraints, cognitive processing, and adjustment to breast cancer. Journal of Consulting and Clinical Psychology, 69(4), $706-711$.

Fisher, L. (2006). Research on the family and chronic disease among adults: Major trends and directions. Families, Systems \& Health, 24(4), 373-380.

Law, D., Crane, D., \& Russell, D. (2000). The influence of marital and family therapy on health care utilization in a health-maintenance organization. Journal of Marital and Family Therapy, 26(3), 281-291.

Lepore, S., Ragan, J., \& Jones, S. (2000). Talking facilitates cognitive-emotional processes of adaptation to an acute stressor. Journal of Personality and Social Psychology, 78(3), 499-508.

McDaniel, S., Hepworth, J., \& Doherty, W. (1992). Medical family therapy: A biopsychosocial approach to families with health problems. New York: Basic Books.

Nijboer, C., Tempelaar, R., Sanderman, R., Triemstra, M., Spruijt, R., \& van den Bos, G. (1998). Cancer and caregiving: The impact on the caregiver's health. Psycho-Oncology, 7(1), 3-13.

Ramsey, C. N. (Ed.). (1989). Family systems in medicine. New York: Guilford.

Rolland, J. (1994). Families, illness, and disability: An integrative treatment model. New York: Basic Books.

Skaff, M., \& Pearlin, L. (1992). Caregiving: Role engulfment and the loss of self. The Gerontologist, 32(5), $656-664$.

Walsh, F., \& Anderson, C. M. (1988). Chronic disorders and the family. New York: Haworth Press.

Weihs, L., Fisher, L., \& Baird, M. (2002). Families, health and behavior: A section of the commissioned report by the Committee on Health and Behavior: Research, Practice and Policy, Division of Neuroscience and Behavioral Health, and Division of Health Promotion and Disease Prevention, Institute of Medicine, National Academy of Sciences. Families, Systems \& Health, 20(1), 7-46. 